\title{
THE JEFFERSON LAB CONTRIBUTION TO THE QCD SPIN STRUCTURE OF THE NUCLEON
}

\author{
M. GARÇON \\ DAPNIA/SPhN, CEA-Saclay, 91191 Gif-sur-Yvette, France \\ E-mail: mgarcon@cea.fr
}

\begin{abstract}
From inclusive to semi-inclusive and exclusive (polarized) deep inelastic scattering, the quark structure of the nucleon is being studied with increasingly precise experiments at Jefferson Lab. We will review here the ongoing programs to determine polarized parton distributions - helicity and transversity - as well as generalized parton distributions, addressing the question of intrinsic spin and orbital angular momentum of quarks in the nucleon.
\end{abstract}

\section{A window into the deep inelastic regime}

With electron beams in the $4-6 \mathrm{GeV}$ energy range, the CEBAF accelerator gives the experimentalist the possibility to probe the nucleon structure in the deep inelastic scattering regime (DIS), here defined by the kinematical condition that the energy $W$ of the $\gamma^{*} p$ system is above most nucleon resonances, typically $2 \mathrm{GeV}$, and the virtuality $Q^{2}$ of the exchanged photon larger than $1 \mathrm{GeV}^{2}$. Though the available range in $W$ is rather modest compared to higher energy machines, it is an interesting finding, developed in this paper, that the (mostly valence) quark structure of the nucleon can be accessed in precise measurements of inclusive, semi-inclusive and exclusive electron scattering.

Figure 1 shows the typical kinematical coverage achieved with a 5.75 $\mathrm{GeV}$ beam and the CEBAF large acceptance spectrometer (CLAS). The higher boundary is due to the beam energy, and to a lesser extent to the electron scattering angle. For $W>2 \mathrm{GeV}$, a significant coverage of the valence quark region $\left(0.15<x_{B}<0.65\right)$ can be obtained, albeit at moderate values of $Q^{2}$. There is however accumulating evidence, from the evolution of parton distributions, from first results on exclusive virtual Compton scattering, and from the description of the $\gamma^{*} \gamma \pi^{0}$ form factor in $e^{+} e^{-}$collisions ${ }^{1}$ with a handbag diagram similar to the one describing the $\gamma^{*} p \rightarrow \gamma^{(*)} p$ reaction, that this reaction can indeed be interpreted in terms of parton distribu- 
tions at $Q^{2}$ as low as 1 or $2 \mathrm{GeV}^{2}$. It is certainly one of the goals of the experiments described here to establish this on a firm ground.

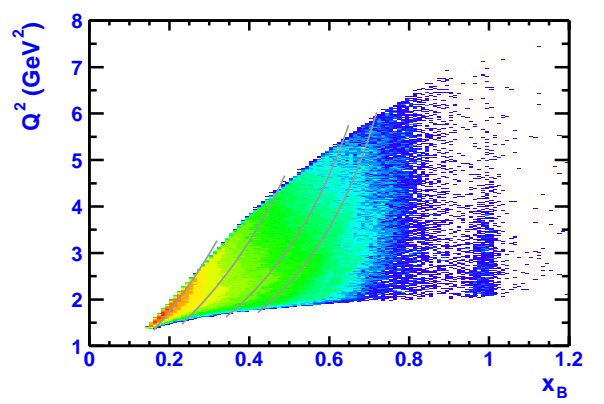

Figure 1. Kinematical coverage for a typical CLAS experiment with a $5.75 \mathrm{GeV}$ electron beam. The lines correspond to constant values of $W(1.8,2,2.4$ and $2.8 \mathrm{GeV}$ from right to left).

The transition from the resonance region to the deep inelastic regime is also studied in great details at JLab, addressing for example the question of higher twists and duality ${ }^{2}$.

\section{Experimental equipment}

One of the main specificities of JLab is the high luminosity, or product luminosity $\times$ acceptance, which can be achieved using the highly polarized electron beam (about $80 \%$ polarization).

Polarized targets are being used in all three experimental Halls. In Hall A, a high density polarized ${ }^{3} \mathrm{He}$ target has been developed, which gives an excellent opportunity to study polarized parton distributions in the neutron. This target is based on optical pumping of Rb atoms, with subsequent spin exchange between $\mathrm{Rb}$ and ${ }^{3} \mathrm{He}$. In Halls B (CLAS) and $\mathrm{C}$, solid longitudinally polarized $\mathrm{NH}_{3}$ or $\mathrm{ND}_{3}$ targets have been installed. Hall $\mathrm{C}$ has in addition the capability of target transverse polarization and is planning on using LiD in coming experiments (deuterated lithium has a higher figure of merit than deuterated ammonium).

In addition to the standard magnetic spectrometers available in the three Halls, dedicated detectors are being used or developed for specific experiments described below. 


\section{Helicity dependent parton distributions}

\subsection{Inclusive scattering}

Using longitudinally polarized electron beam incident upon a longitudinally $(/ /)$ or transversely $(\perp)$ polarized target, double spin asymmetries are measured in inclusive $\overrightarrow{e^{\prime}} \vec{N} \rightarrow e^{\prime} X$ scattering. The asymmetries $A_{/ /}$and $A_{\perp}$ with respect to the beam direction are translated into asymmetries $A_{1}$ and $A_{2}$ with respect to the virtual photon direction. These in turn, using the known unpolarized structure functions appearing in their denominator, determine the two polarized structure functions $g_{1}\left(x, Q^{2}\right)$ and $g_{2}\left(x, Q^{2}\right)$. In the quark parton model, $g_{1}$ has the well-known probability interpretation in the Bjorken regime $\left(Q^{2} \rightarrow \infty\right)$ :

$$
g_{1}(x)=\frac{1}{2} \sum_{q, \bar{q}} e_{q}^{2}\left[q^{\uparrow}(x)-q^{\downarrow}(x)\right]=\frac{1}{2} \sum_{q, \bar{q}} e_{q}^{2} \Delta q(x)
$$

It measures the contribution of the quark intrinsic spin to the nucleon spin. $g_{2}$ represents interaction beyond the quark parton model, and as such, is a good tool to study higher twist effects and is sensitive to quark-gluon correlations.

The behaviour of these asymmetries and polarized structure functions is hardly explored at high values of $x$. Yet, it could lead to significant constraints on the valence quark distributions, whose $x$-dependence is often parameterized as $x \rightarrow 1$; it is useful to calculate (high) moments of these distributions to be compared directly with lattice QCD calculations; and it poses interesting theoretical problems as the QCD factorization and evolution may break down at the limit of very high $x^{3}$.

It is an experimental challenge to perform such measurement, since both the Mott cross section and the unpolarized quark distributions $q(x)$ decrease as $x$ increases.

New results on the proton and deuteron asymmetries $\left(A_{1 p}\right.$ and $\left.A_{1 d}\right)$ from CLAS ${ }^{4}$ were shown at this conference and will soon be available . The neutron asymmetry $A_{1 n}$ has recently been measured in Hall A ${ }^{5}$. Three precise data points at $0.32<x<0.6$ give the first clean signature of positive $A_{1 n}$ at high $x$. The trend deviates from PQCD predictions based on hadron helicity conservation, which states a quark with $x \rightarrow 1$ has the same helicity as the nucleon. This finding points to the role of quark orbital angular momentum in the nucleon.

Using parametrizations of unpolarized structure functions $F_{1}$ and world data for $g_{1}^{p} / F_{1}^{p}$, neglecting strangeness at high $x$, the quantities $(\Delta u+$ 
$\Delta \bar{u}) /(u+\bar{u})$ and $(\Delta d+\Delta \bar{d}) /(d+\bar{d})$ were extracted ${ }^{5}$. Since the difference $(\Delta q+\Delta \bar{q}) /(q+\bar{q})-\Delta q_{v} / q_{v}$, is smaller than experimental uncertainties, the polarized valence quark distributions $\Delta q_{v}$ may be determined from these ratios (see Figure 2). This new measurement constrains especially $\Delta d_{v}$.
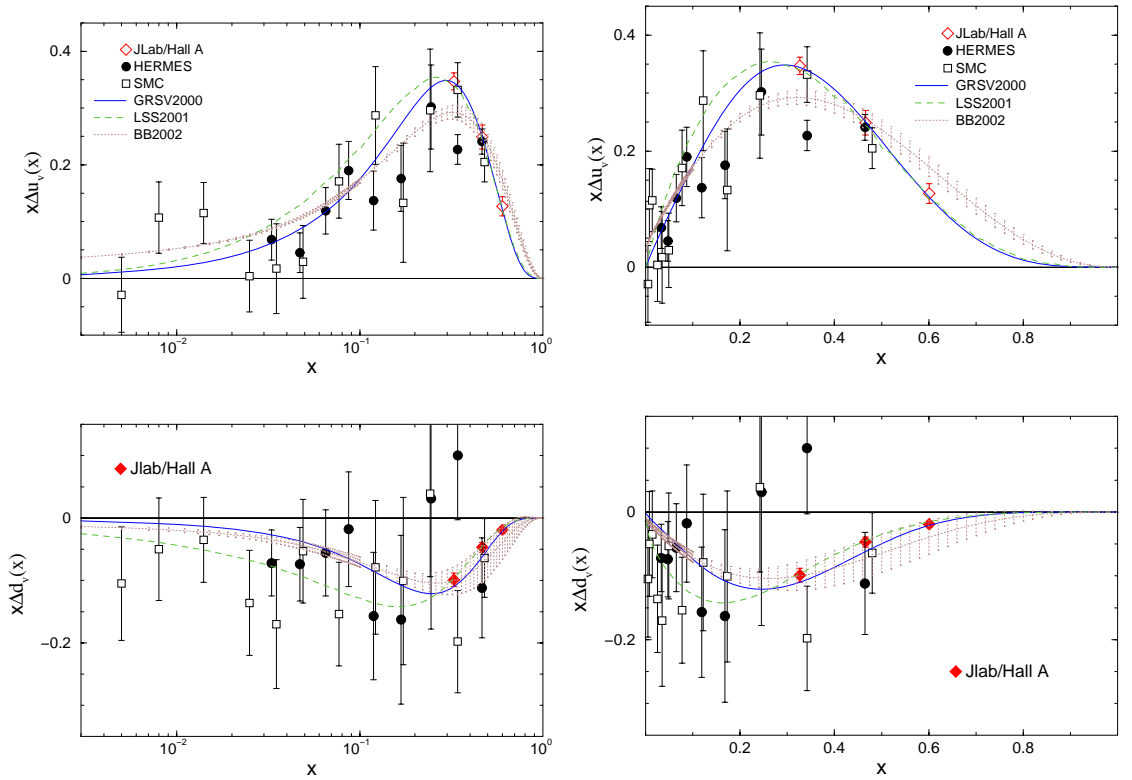

Figure 2. Polarized valence quark distributions in the nucleon calculated using the JLab/Hall A results ${ }^{5}$. Adapted from Figure 16.5 of Ref. ${ }^{6}$, which contains all references to previous experiments and parameterizations. $x$-scale is logarithmic on the left, linear on the right.

Even higher values of $x$ will be accessible with the planned $12 \mathrm{GeV}$ upgrade of the CEBAF accelerator ${ }^{7}$ and/or using measurements in the resonance region provided quark-hadron duality is established ${ }^{2}$.

\subsection{Semi-inclusive scattering (SIDIS) and flavor decomposition}

Detecting the hadron from the current quark fragmentation in the semiinclusive process $\vec{e} \vec{N} \rightarrow e h X$, a double spin asymmetry $A_{/ /}^{h}$ can be measured, using both longitudinally polarized beam and target. Assuming leading order $x-z$ factorization, one gets for each detected hadron species 
$h$ an asymmetry

$$
A_{1 N}^{h}\left(x, Q^{2}, z\right) \equiv \frac{\Delta \sigma^{h}\left(x, Q^{2}, z\right)}{\sigma^{h}\left(x, Q^{2}, z\right)}=\frac{\sum_{q} e_{q}^{2} \Delta q\left(x, Q^{2}\right) \cdot D_{q}^{h}\left(z, Q^{2}\right)}{\sum_{q} e_{q}^{2} q\left(x, Q^{2}\right) \cdot D_{q}^{h}\left(z, Q^{2}\right)}
$$

where $D_{q}^{h}$ describes the fragmentation of the quark $q$ in a hadron $h$ of energy $z \times$ the virtual photon energy.

The so-called "Semi-SANE" program ${ }^{8}$ is to measure eight such asymmetries, for $h=\pi^{ \pm}$and $K^{ \pm}$, for both proton and deuteron $\left(\mathrm{NH}_{3}\right.$ and $\mathrm{LiD}$ polarized targets) in Hall C. Together with the two inclusive asymmetries $A_{1 N}$, this will lead to the extraction of five polarized quark distributions $\Delta u, \Delta \bar{u}, \Delta d, \Delta \bar{d}$ and $\Delta s$. The originality of this experiment will be the use of a non-magnetic spectrometer in direct view of the target to detect the scattered electrons. The Big Electron Telescope Array (BETA), under construction, will consist of a 1744 blocks lead-glass calorimeter, a gas Cerenkov detector for pion rejection and a lucite hodoscope array for tracking and redundant particle identification. It will cover a solid angle close to $200 \mathrm{msr}$. In conjunction with the standard Hall C HMS spectrometer also equiped with gas Cerenkov and aerogel detectors for $\pi / K$ idendification, luminosities of the order of $10^{35} \mathrm{~cm}^{-2} \mathrm{~s}^{-1}$ should be achieved. Anticipated results were discussed at this conference ${ }^{8}$.

\section{Tests of factorization in SIDIS}

At the relatively low energies used at JLab for these experiments, one could question the validity of the (leading order) $x-z$ factorization, resulting in Eq. (2). There is increasing evidence that this factorization is not, or not much, violated at $6 \mathrm{GeV}$. For example, cross section for the $H\left(e, e^{\prime} \pi^{-}\right) X$ reaction measured in Hall $\mathrm{C}$ are in excellent agreement with Monte-Carlo based on LO $x-z$ factorization ${ }^{9}$. Also semi-inclusive asymmetries $A_{1 p}^{\pi^{+}}$ measured with CLAS agree with HERMES and SMC data and fall on the same curve as the inclusive $A_{1 p}$; within experimental errors, no $z$ dependence is observed ${ }^{10}$.

\section{Transversity}

In SIDIS, at leading order, eight response functions characterize the distribution of cross section. Three contributions are associated with a transversely polarized target, two of which should be measurable. The transverse nucleon polarization 
- affects the quark transverse momentum: this asymmetric $k_{\perp}$ distribution, combined with the usual fragmentation function $D$, gives rise to the Sivers effect, characterized by a $\sin \left(\phi_{h}-\phi_{S}\right)$ dependence in the $\vec{N}\left(e, e^{\prime} h\right) X$ cross section.

- induces quark transverse polarization: this may manifest itself through the Collins fragmentation function which describes the correlation of the outgoing hadron (transverse) momentum with the quark transverse polarization; this effect is characterized by a $\sin \left(\phi_{h}+\phi_{S}\right)$ dependence.

The $\phi$ angles refer here to the final hadron $(h)$ and target spin $(S)$ azimuthal angles with respect to the electron scattering plane. Both effects are the subject of intense studies. The Collins effect is of particular interest, since its measurement would lead to the determination of the transversity distribution ${ }^{11}$. Let us recall that the transversity and helicity distributions are identical in a non relativistic quark model picture of the nucleon, and that transversity does not mix with gluons in its evolution and is suppressed at low $x$ with respect to $\Delta q(x)$. Finally, its first moment yields the nucleon "tensor charge" $\delta q$ calculable in lattice QCD.

Using the polarized ${ }^{3} \mathrm{He}$ target and the newly installed Bigbite detector in Hall A, a dedicated high precision experiment ${ }^{12}$ will desentangle these two effects, measure the transversity distribution in the neutron (sensitive to $\delta d$ ) and probe other $k_{\perp}$-dependent quark distribution functions. Such single spin asymmetries measurements using a transversely polarized ${ }^{3} \mathrm{He}$ target are complementary to HERMES and COMPASS transversity measurements, respectively on the proton and on the deuteron, and are performed at $x$ values where transversity might be larger.

\section{Other single spin asymmetries (SSA)}

There is a rich phenomenology associated with various SSA linked to transverse momentum distributions of partons, and to orbital angular momentum. One example is the determination of the scalar distribution $e_{q}(x)^{13}$. On the experimental side, the consistency (through a model) between HERMES and CLAS results ${ }^{10,14}$ on $A_{L U}$ and $A_{U L}$ measurements (using either polarized beam or target) may be interpreted as a further indication of the applicability of factorization. Definitive conclusions on the measurability of several quark transverse momentum distributions require more data and higher accuracy, in order to disentangle various azimuthal dependences as well as distinguish between twist-2 and twist-3 contributions. JLab is also 
pursuing this path.

\section{Generalized Parton Distributions}

Generalized Parton Distributions (GPDs) have emerged as an attractive, powerful and unifying concept for the nucleon structure ${ }^{15}$. Besides offering a link between "ordinary" parton distributions and elastic form factors, they contain the interferences, or coherences, between states of different parton longitudinal momentum fractions and they include correlations between transverse position and longitudinal momentum. Last but not least, their determination could yield the total angular momentum (intrinsic spin + orbital angular momentum) carried by the quarks in the nucleon. Recent theoretical progress include lattice QCD calculations of GPD moments ${ }^{17}$ and refined phenomenological analyses of the link with form factors ${ }^{18}$.

GPDs enter the leading order description of deeply exclusive leptoproduction of photons - DVCS - and of mesons (see e.g. Ref. ${ }^{16}$ ).

\subsection{Deeply virtual Compton scattering (DVCS)}

After the first observation of DVCS at H1 (in the gluon sector) and at CLAS and HERMES, preliminary CLAS results obtained at 4.8 and $5.75 \mathrm{GeV}$ were shown in this talk. Increased statistics allow for a finer binning (in $Q^{2}, x$ and $t$ ), but the separation between $e p \rightarrow e p \gamma$ and $e p \rightarrow e p \pi^{0}$ reactions is increasingly difficult. Dedicated DVCS experiments at JLab will detect all three particles in the final state. Their primary objective is to establish on firm grounds the link between observables and GPDs. This goes through the study of characteristic scaling laws in observables. If scaling laws are manifest, or deviations thereof understood, then these measurements will put stringent constraints on GPD models or parameterizations.

The dedicated Hall A experiments on $\overrightarrow{e p} \rightarrow e p \gamma^{19}$ and $\overrightarrow{e n} \rightarrow e n \gamma^{20}$ (quasi-free reaction on a deuterium target) were completed in the Fall 2004. The CLAS/DVCS experiment ${ }^{21}$ will run in the Spring 2005. The observable being measured is the beam spin cross section difference, which originates from the interference between the DVCS (photon emission from the nucleon) and the Bethe-Heitler (photon emission from the incident or scattered electron) processes:

$$
\Delta \sigma=\sigma^{+}-\sigma^{-}=\Gamma \cdot(\mathcal{A} \sin \phi+\ldots)
$$

or the beam spin asymmetry $\Delta \sigma /(2 \sigma)$, where $\Gamma$ is a known factor and

$$
\mathcal{A}=F_{1}(t) \cdot \mathcal{H}+\frac{x_{B}}{2-x_{B}}\left[F_{1}(t)+F_{2}(t)\right] \cdot \tilde{\mathcal{H}}-\frac{t}{4 M^{2}} F_{2}(t) \cdot \mathcal{E} .
$$


Here $F_{1}$ and $F_{2}$ are the elastic nucleon form factors (originating from the Bethe-Heitler amplitude) while the GPDs $H^{q}, \tilde{H}^{q}$ and $E^{q}$ enter the imaginary part of the DVCS amplitude through $\mathcal{H}=$ $\pi \sum_{q} e_{q}^{2}\left[H^{q}(\xi, \xi, t) \pm H^{q}(-\xi, \xi, t)\right]$ and similar expression for $\tilde{\mathcal{H}}$ and $\mathcal{E}$. Note that the dominant term in Eq. (4) is the first one for DVCS on the proton, while the last term dominates in the case of the neutron. The neutron experiment has then the potential to access the less constrained GPD $E$ and thus yield information on the quark orbital angular momentum. While the $\sin \phi$ dependance in Eq. (3) originates from the helicity conserving leading twist contributions, the additional terms will be determined as well and provide a quantitative measure of higher-twist contributions.

For these experiments, new compact electromagnetic calorimeters were developed $\left(\mathrm{PbF}_{2}\right.$ in Hall $\mathrm{A}$, equiped with fast digitizing electronics, $\mathrm{PbWO}_{4}$ in CLAS, in conjunction with a background sweeping supraconducting solenoid) for the detection of the photons originating from DVCS.

\subsection{Hard exclusive meson electroproduction}

Deeply virtual vector meson production is complementary to DVCS since it could give access to the flavor separated $H$ and $E$ GPDs. The contribution to observables from the longitudinal photons must be isolated and one must reach higher $Q^{2}$ since the additional gluon exchange necessary to produce the meson increases the higher-twist contributions.

Longitudinal cross sections have been extracted for $\rho$ electroproduction at $4.2 \mathrm{GeV}$ and are in qualitative agreement with a model based on GPDs ${ }^{22}$. For the $\omega$ channel however, recent CLAS data at $5.75 \mathrm{GeV}$ show that the handbag diagram does not dominate ${ }^{23}$. This is specific to the case of the $\omega$, as the $t$-channel $\pi^{0}$ exchange was shown to dominate the cross section, even at relatively high $Q^{2}$.

\section{Conclusions}

Inclusive and semi-inclusive deep inelastic scattering is being understood at moderate values of $Q^{2}$, yielding information about the quark structure of the nucleon. Factorization is shown to work at lower energies than previously thought. Jefferson Lab, with the CEBAF beam energy nearing 6 $\mathrm{GeV}$ for the past two years, contributes and will contribute fully to the determination of polarized quark distributions (valence helicity distributions were presented, and transversity measurements are being prepared). In addition, a rich phenomenology in quark transverse momentum distributions 
is being explored experimentally through single spin asymmetries.

JLab, with its high luminosity and high resolution detectors, is especially well equiped for the measurement of deep exclusive reactions. Once proper scaling laws are verified for DVCS (and possibly for hard meson electroproduction), significant constraints on the generalized parton distributions will be set by new dedicated experiments. A complete mapping and measurement of GPDs will probably have to await the planned $12 \mathrm{GeV}$ upgrade ${ }^{7}$ of the CEBAF accelerator and of the Halls detectors.

\section{References}

1. J. Gronberg et al. (CLEO collaboration), Phys. Rev. D57, 33 (1998).

2. R. De Vita, these proceedings.

3. X. Ji, Proc. of the HiX2004 Workshop on the Structure of the Nucleon at Large Bjorken $x$, Marseille (2004), to be published; W. Vogelsang, ibidem.

4. G. Dodge, S. Kuhn, M. Taiuti et al.(CLAS collaboration), private communication.

5. X. Zheng et al., Phys. Rev. Lett. 92, 012004 (2004); and Phys. Rev. C70, 065207 (2004).

6. Particle Data Group, Phys. Lett. B592, 1 (2004).

7. Preliminary Conceptual Design Report, http://www.jlab.org/div_dept/physics_division/GeV/index.html

8. X. Jiang, contribution to this conference.

9. R. Ent, private communication.

10. H. Avakian (CLAS collaboration), private communications.

11. V. Barone, these proceedings.

12. J.P. Chen, X. Jiang, J.C. Peng et al., CEBAF experiment E03-004.

13. A.V. Efremov et al., Phys. Rev. D64, 114014 (2003); M. Wakamatsu, contribution to this conference.

14. H. Avakian et al., Phys. Rev. D69, 112004 (2004).

15. X. Ji, Phys. Rev. Lett. 78, 610 (1997) and Phys. Rev.D55, 7114 (1997); A. Radyushkin, At the Frontier of Particle Physics / Handbook of QCD, M. Shifman ed., World Scientific, Singapore (2001) [hep-ph/0101225].

16. M. Garçon, Eur. Phys. J. A 18, 389 (2003); and references therein.

17. M. Gockeler et al. (QCDSF Collaboration), hep-lat/0410023.

18. M. Diehl et al., hep-ph/0408173; M. Guidal et al., hep-ph/0410251.

19. P. Bertin, C. Hyde-Wright, R. Ransome, F. Sabatié et al., CEBAF experiment 00-110.

20. P. Bertin, C. Hyde-Wright, F. Sabatié, E. Voutier et al., CEBAF experiment 03-106.

21. V. Burkert, L. Elouadrhiri, M. Garçon, S. Stepanyan et al., CEBAF experiment 01-113.

22. C. Hadjidakis et al. (CLAS collaboration), hep-ex/0408005.

23. M. Garçon et al. 10th Int. Symp. on Meson-Nucleon Physics and the Nucleon Structure, Beijing (2004). 